Regular article

\title{
Resting heart rate estimation using PIR sensors
}

\author{
Hemanth Kapu ${ }^{a}$, Kavisha Saraswat ${ }^{a}$, Yusuf Ozturk ${ }^{a, *}$, A. Enis Cetin ${ }^{\text {b,c }}$ \\ ${ }^{a}$ Department of Electrical and Computer Engineering, San Diego State University, San Diego 92115, CA, USA \\ ${ }^{\mathrm{b}}$ Department of Electrical and Computer Engineering, University of Illinois, Chicago 60607, USA \\ ${ }^{\mathrm{c}}$ Department of Electrical and Electronics Engineering, Bilkent University, Ankara 06800, Turkey
}

\section{H I G H L I G H T S}

- A non-invasive and non-contact system for estimating resting heart rate (RHR) is developed using a pyroelectric infrared (PIR) sensor.

- This new system provides a low cost and an effective way to estimate the resting heart rate, which is an important biological marker.

- Data is collected using PIR sensors placed on a table $1 \mathrm{~m}$ from a subject sitting on a chair in regular daily clothing. Output of the PIR sensor is sampled with a sampling rate of $10 \mathrm{~Hz}$.

- The second-order derivative of the discrete-time PIR sensor is computed to extract the heart beat signal.

- A total of 30 subjects ( 5 females) are recruited. A total of 60 experiments were conducted, collecting over 10,000 heart beats.

- A medical grade PPG sensor is used for validation of the ambient sensor.

- Experimental results showed that $95 \%$ of the estimated heart rate values are within 4 beats per minute.

- Our results further show that the average values of the 60 RHR (30 subjects, two measurements per subject) measurements using the PPG and the PIR sensors are 75.5 beats per minute and 74.7 beats per minute, respectively.

\section{A R T I C L E I N F O}

\section{Article history:}

Received 22 February 2017

Revised 15 March 2017

Accepted 15 May 2017

Available online 28 May 2017

\section{Keywords:}

Non-contact system

Resting heart rate

PIR sensor

\begin{abstract}
A B S T R A C T
In this paper, we describe a non-invasive and non-contact system of estimating resting heart rate (RHR) using a pyroelectric infrared (PIR) sensor. This infrared system monitors and records the chest motion of a subject using the analog output signal of the PIR sensor. The analog output signal represents the composite motion due to inhale-exhale process with magnitude much larger than the minute vibrations of heartbeat. Since the acceleration of the heart activity is much faster than breathing the second derivative of the PIR sensor signal monitoring the chest of the subject is used to estimate the resting heart rate. Experimental results indicate that this ambient sensor can measure resting heart rate with a chisquare significance level of $\alpha=0.05$ compared to an industry standard PPG sensor. This new system provides a low cost and an effective way to estimate the resting heart rate, which is an important biological marker.
\end{abstract}

(c) 2017 Elsevier B.V. All rights reserved.

\section{Introduction}

The heart has been considered the source of courage, emotion, and wisdom for centuries [1]. Essentially, it appeared that the heart could affect our awareness, perceptions and intelligence. Numerous studies have since shown that heart coherence is an optimal physiological state associated with increased cognitive function, self-regulatory capacity, emotional stability and resilience [2].

Over the years, many studies have been conducted that utilized various physiological measures such as electroencephalogram (EEG) $[3,4]$, skin conductance level or galvanic skin response

\footnotetext{
* Corresponding author.

E-mail address: yozturk@mail.sdsu.edu (Y. Ozturk).
}

(SCL/GSR) [5], electrocardiogram (ECG) [6], blood volume pressure (BVP) [7] or combination of two or more physiological markers $[8,9]$ to monitor human well-being and state of mind. However, it was the heart rate variability, or heart rhythms that stood out as the most dynamic and reflective indicator of oneś emotional state and healthy well-being [10,11].

Resting Heart Rate (RHR) is one of the most important biomarkers indicating the health condition of a subject $[10,12]$. RHR is the number of heart beats per minute while the subject is at rest. Measuring RHR gives real-time preview of how the heart muscles are functioning. RHR, when considered in the context of other markers, such as blood pressure and cholesterol, can help identify potential health problems. Usually, lower the RHR, higher the level of physical fitness and vice versa. High RHR could be an indicator of ele- 
vated probability of cardiac uncertainty. In some cases a low RHR may result occasional giddiness and fatigue.

Accurate measurement and monitoring of physiological parameters play an important role in a broad range of applications in the field of healthcare, psycho-physiological examinations and sports training. Wearable methods for the measurement of physiological parameters are dependent on the sensors to be attached to a subject, such as electrocardiogram (ECG) [13], pulse oximetry [14], piezoelectric transducer [15], and so on. The issue with such contact-based methods is that it may cause undesirable skin irritation, discomfort and soreness to the subject. Currently, laser doppler [16], microwave doppler radar [17], ultra-wideband radar [18], and frequency modulated continuous wave radar [19] systems are being investigated for contact-free measurements of physiological parameters. However, these systems require expensive hardware. Consequently, demand for low-cost and convenient non-contact methods of measuring and monitoring physiological signals has grown inquisitively.

In this paper, we describe a novel system capable of noncontact measurement of RHR. The system estimates the RHR from the chest motion of the subject using a pyroelectric infrared (PIR) sensor [20]. PIR sensors are small, inexpensive, low power, rugged, are easy to interface with, and are easy to use. One of their best features is that they do not wear out. PIR sensors are mostly employed as occupancy detectors [21]. They have been extensively used in motion tracking systems. PIR sensors having digital output and the modulated visibility of fresnel lenses can provide capabilities for tracking human motion [22], and counting people crossing door of a room or building. However, the analog output signal of PIR sensors can provide beyond simple presence of a subject, including the instantaneous motion of the body [23].

Our design detects the change in infrared thermal heat patterns in front of a sensor to estimate the RHR. The sensor uses a couple of pyroelectric elements that respond to temperature variations within the viewing zone. Instantaneous differences in the output of the two elements are detected as motion, especially the motion of a heat-bearing object, such as a human. To the best of our knowledge, this is the first paper that demonstrates the feasibility of heart rate detection using a PIR sensor.

The paper introduces a new methodology for extracting RHR from IR radiations off the human body. An algorithm is presented that captures mathematical formulation of the problem, and is experimentally shown to perform well in practice. The paper also presents an empirical comparison with PPG sensor readings.

The rest of the paper is organized as follows: Section 2 discusses the background of this method and related past work in this field. Section 3 explains the methodology of this system and data collection procedure. Section 4 presents the experimental results of the system. Finally, Section 5 offers concluding remarks.

\section{Background and related work}

Over the last 20 years, heart rate monitors (HRMs) have become a widely used aid for a variety of applications [11]. The development of new HRMs has also evolved rapidly during the last two decades. In addition to heart rate (HR) responses, research has recently focused more on heart rate variability (HRV). Existing approaches for extracting heart-related signals fall under two categories: wearable sensor technologies [24] and non-contact (ambient) techniques [25-36].

The traditional method for monitoring heart rate measured the spikes of the potential generated by heart at each pressure pulse i.e. electrocardiogram, or ECG [9]. Physiological measurements, such as ECG signals, are robust because they are controlled by involuntary activations of the autonomic nervous system (ANS).
However, existing sensors that can extract these signals require physical contact with subjects body, and causes interference with the user experience. Various wearable technologies, for instance, a portable ECG device, worn as a chest band holds the sensor centered over the heart, may transfer the readings wirelessly to the host computer. Unfortunately, the chest band is uncomfortable when worn for extended periods. It has a role to play in medical and exercise settings, but it is not a viable option for continuous use. In a similar fashion, wristband or a smart watch have shortcomings [24]. The devices operation can be compromised by: movement of body, for instance, gesturing, exercises based on arm motion that can alter the blood circulation at wrist; and the displacement of wearable device across the skin surface. Despite the higher reliability and good signal quality of fixed-on-body electrodes, they are inconvenient and inadequate for long-term and everyday measurements. The presence of cables (one for each of the electrodes placed) can limit the patient mobility and comfort to an extent, forcing him to maintain the position for all the monitoring period of time.

Non-contact techniques, on the other hand, are non-intrusive and more adequate for long-term monitoring. Despite some shortcomings, the non-intrusive nature makes them an attractive option for daily monitoring. Several techniques based on laser doppler [16], microwave doppler radar [17], ultra-wideband radar [18], frequency modulated continuous wave radar [19] and thermal imaging are investigated. The advantage of these approaches is that they do not require users to wear any sensors on their bodies. However, as they rely on outwardly expressed states, they tend to get interfered with external factors that are, however, easily controlled or suppressed. In contrast, our system can capture physiological signals without requiring the user to wear any sensors by relying purely on wireless signals reflected off her/his body. Our work is closest to prior work that uses RF signals [37] to extract subject's breathing rate and RHR.

Our wireless vital sign monitor measures heart rate by applying signal processing technique to collected cardiorespiratory data. When measuring the vital signs with a non-contact monitor, available monitors detect both respiration and heartbeat. The larger magnitude of respiration signal typically overshadows the smaller heartbeat. This system uses a computing algorithm to determine the heart rate from the chest movements recorded using PIR Sensor.

\section{Methodology}

\subsection{PIR sensors}

PIR sensors are widely used for sensing motion of subjects. Infrared radiation exists in the electromagnetic spectrum at a wavelength that is longer than visible light [21]. Objects that generate heat also generate infrared radiation and those objects include animals and the human body. PIR sensors are made of pyroelectric sensors that can detect the levels of infrared radiations and thus are used commercially used for automation of electrical appliances and home surveillance systems.

The basic functionality of differential PIR sensor is to measure the difference in infrared radiation density of two pyro-electric elements within the sensor. Normal variations in the temperature caused by the air are nullified by the two elements connected in parallel. If the elements measure same amount of infrared radiations, the sensor produces zero output. Most of the commercially available PIR motion sensor circuits produce digital output. Nonetheless, analog signal output can also be obtained from PIR sensors. We exploit the analog signal obtained from the PIR sensor 
to detect chest motion and eventually help estimate resting heart rate using zero crossing.

\subsection{Data acquisition}

The data is collected using PIR sensors as shown in Fig. 1. The analog output of the PIR sensor is sampled with a sampling rate of $10 \mathrm{~Hz}$, using a microcontroller and the resulting signal is fed to a personal computer.

The second-order derivative of the discrete-time PIR sensor is computed to extract the heart beat signal. Zero crossing algorithm is then applied to the resultant output to estimate the resting heart rate.

The PIR sensor detects the chest motion, caused by the inhaleexhale process and the resting heart rate, to provide an analog signal. The chest motion is a resultant of two physiological processes: respiration and heartbeat vibrations [37]. The respiratory activity is, however, much larger in magnitude in comparison to the heartbeat vibrations. On the other hand the acceleration of the breathing activity is much lower than the acceleration of the heartbeat vibrations.

The output signal obtained from the PIR sensor is essentially due to the motion of the chest motion. Computing the secondorder derivative of the signal with respect to time provides us a result related with the acceleration of the chest. As a result the second order derivative signal must be mainly due to the heartbeat activity.

The impulse response of the most widely used first-derivative filter is

$h[n]=\left[\begin{array}{lll}1 & 0 & -1\end{array}\right]$

The corresponding transfer function is $H(z)=z+z^{-1}$.

By convolving this filter with itself, we get the second-order derivative filter,

$h_{2}[n]=\left[\begin{array}{lllll}1 & 0 & -2 & 0 & 1\end{array}\right]$

However the filter $h_{2}[n]$ cannot be used to estimate the resting heart rate, because the recorded data from the PIR Sensor, is noisy. Therefore, the data should be smoothed with a simple Lagrange low pass filter with an impulse response of [121] before applying the second-order derivative filter. Since the sampling frequency of the PIR signal is $10 \mathrm{~Hz}$, our full band is $5 \mathrm{~Hz}$. The LPF is an approximate half-band filter, i.e., it attenuates the high frequency compo- nents above $2.5 \mathrm{~Hz}$. Therefore it does not affect the RHR of a person. It removes some small ripples in the signal. The main effect of the LPF is to remove small ripples in the signal which may be due to $\mathrm{A} / \mathrm{D}$ conversion.

The equivalent impulse response becomes

$g_{2}[n]=\left[\begin{array}{lllllll}1 & 2 & -1 & -4 & -1 & 2 & 1\end{array}\right]$

This is the filter used by [37] to compute the second-order derivative of an $\mathrm{RF}$ signal to extract the heart signal.

Since the Lagrange low-pass filter [121] is also a triangular window, it is possible to scale the window size before applying the second-order derivative filter $h_{2}[n]$.

We observed that convolving the data with a wider triangular window [14641] provides better noise cancellation. Consequently, the effective impulse response of our filter becomes

$$
g_{2}^{\prime}[n]=h_{2}[n] *\left[\begin{array}{lllll}
1 & 4 & 6 & 4 & 1
\end{array}\right]
$$

or

$$
g_{2}^{\prime}[n]=\left[\begin{array}{lllllllll}
1 & 4 & 4 & -4 & -10 & -4 & 4 & 4 & 1
\end{array}\right]
$$

Normalized version of the corresponding input/output relationship is given by

$$
\begin{aligned}
y[n]= & (10 x[n]+4(x[n-1]+x[n+1])-4(x[n-2]+x[n+2]) \\
& -4(x[n-3]+x[n+3])-(x[n-4]+x[n+4])) / 36
\end{aligned}
$$

In our observation, the filter given in Eq. (6) produces a better result than the filter given by Eq. (3). In fact, the filter given in Eq. (6) is also related with one of the filters proposed in [38]. The computational load of the heart-rate estimation algorithm is low because the FIR filter given in Eq. (6) has integer coefficients. Therefore the overall system can be implemented using a low cost digital signal processor or a micro-controller.

\section{Experimental results}

\subsection{Experimental setup}

A total of 30 subjects ( 5 females) are recruited. The age group of subjects lies between 20 and 55 years old. During the experiment subjects were wearing their own outfits. The experiment was conducted in a standard lab environment. The lab environment contains furniture which includes tables, desks, chairs and

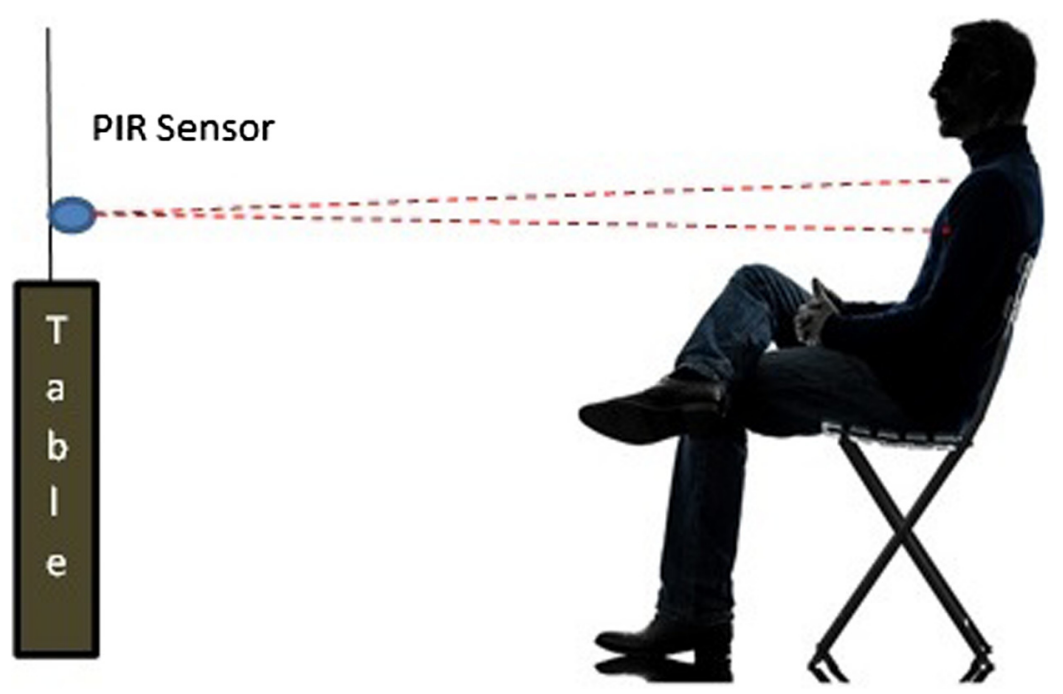

Fig. 1. Data collection experimental setup. 

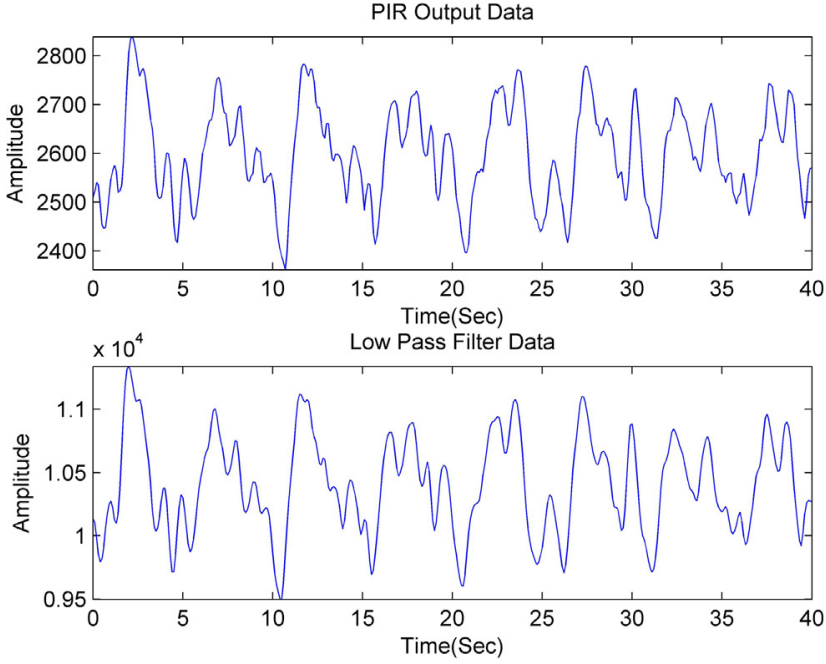

Fig. 2. PIR sensor signal due to chest motion (top) and its low-pass filtered version (bottom).

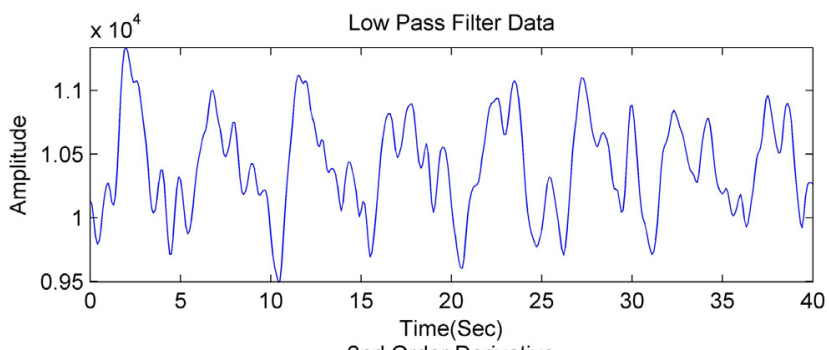

2nd Order Derivative

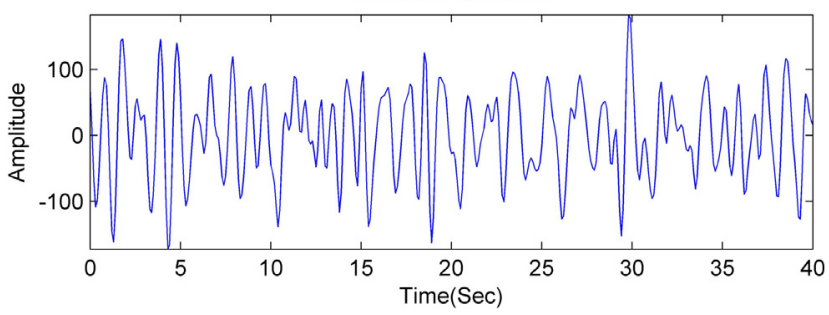

Fig. 3. Filtered output and the corresponding 2nd order derivative signal (bottom). computers. During the experiment PIR sensor is placed on the desk $1 \mathrm{~m}$ away from subject. The subject is instructed to breath in and out as he/she does regularly, during which the chest movements are captured. Prior to the experiment an institutional review board (IRB) approval is obtained from human research protection program (HRPP). The results are compared to the industry standard photoplethysmogram (PPG) sensor and results shows that the estimated values are matching with the photoplethysmogram (PPG) sensor.

Fig. 1 shows the experimental setup how the data is collected. PIR sensor is placed on the stand in front of subject for data collection.

Fig. 2 shows the PIR sensor signal output due to chest motion of one of the subjects who is about $1 \mathrm{~m}$ away from the sensor and the output after low pass filtering. Fig. 3 shows the output of the 2nd order derivative filter and Fig. 4 shows zero crossings in the output signal. By counting the zero crossings of the 2 nd order derivative signal per minute we estimate the RHR. Since this person has 20 zero crossings in $10 \mathrm{~s}$ his RHR is 60 beats per minute.

\subsection{Accuracy in comparison to photoplethysmogram (PPG) sensor}

A total of 60 experiments were conducted, collecting over 10,000 heart beats. During the experiment each subject is simultaneously monitored with a PIR sensor and a PPG device. The estimated RHR values and the industry standard RHR values obtained using the PPG sensor are shown in Fig. 5, which was obtained from 30 subjects. From each subject two PIR records were collected. The horizontal axis represents the experiment number and vertical axis represents the heart rate in BPM (beats per minute). Fig. 5 shows the scatter plot between heart rate values of the PPG device and the PIR sensor, where $x$-axis represents heart rate values of the PPG device and $y$-axis represents heart rate values of the PIR sensor. There is a strong correlation between the two sensors.

Fig. 6 shows the cumulative distribution function (CDF) of the difference between estimated PIR sensor values and the industry standard PPG sensor. The cumulative distribution function (CDF) shows that the $95 \%$ of values have deviation less than 4 beats per minute. Our results further show that the mean values of the 60 RHR (30 subjects, two measurements per subject) measure-

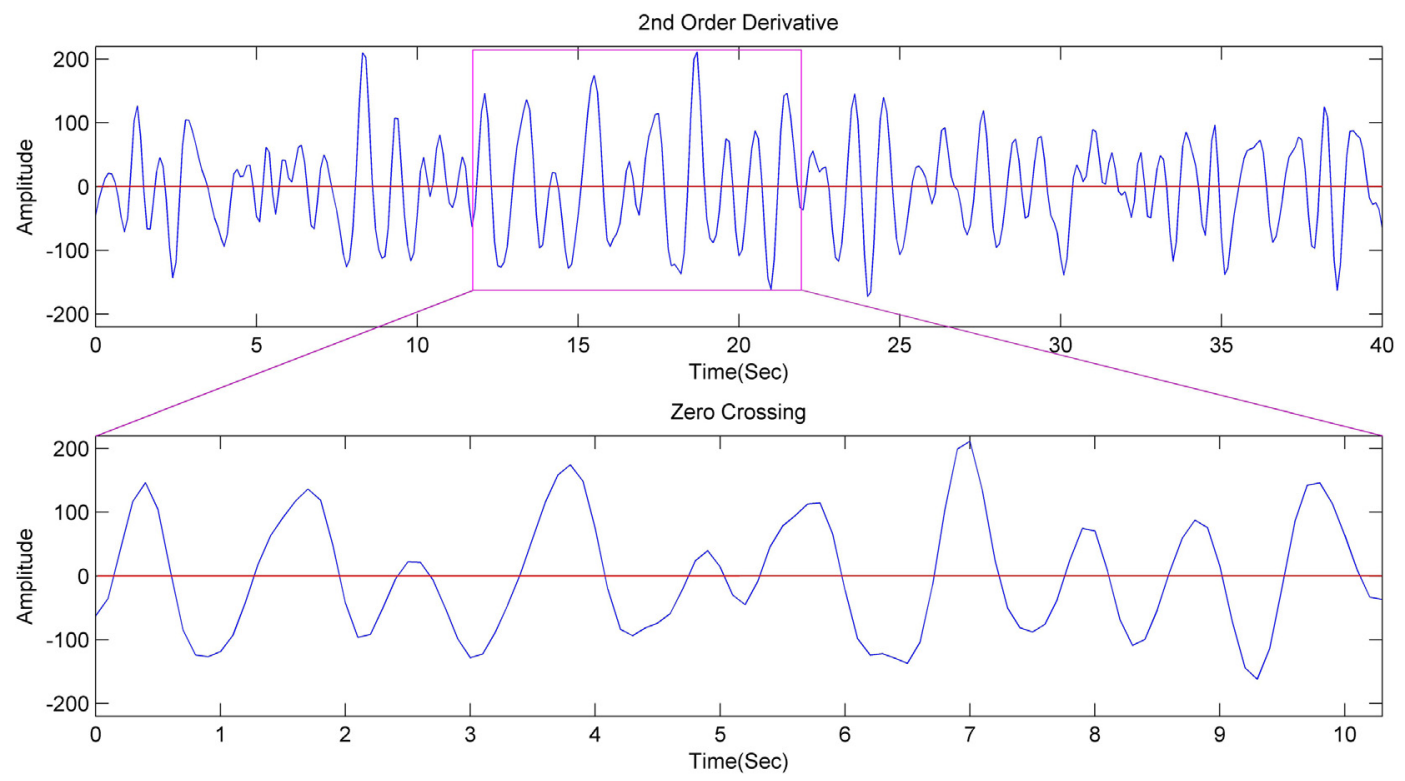

Fig. 4. Zero crossing from the second order derivative signal. 


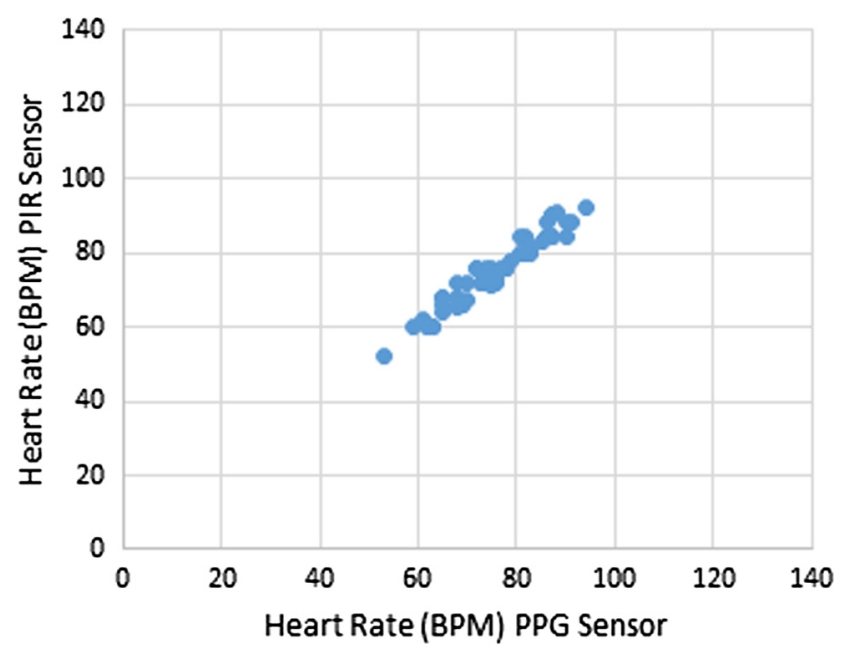

Fig. 5. Scatter plot of the PIR sensor and the industry standard PPG sensor RHR values.

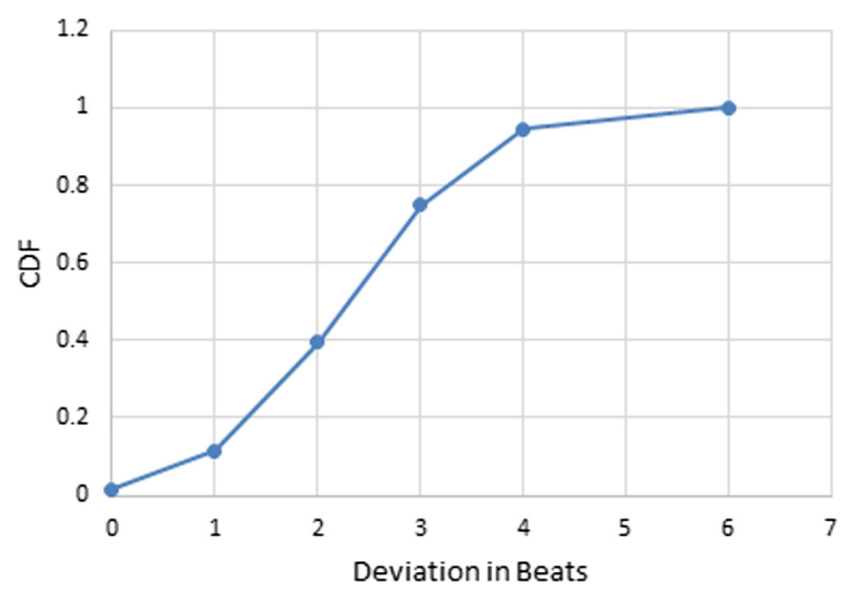

Fig. 6. CDF of deviation in beats.

ments using the PPG and the PIR sensors are 75.5 BPM (beats per minute) and 74.7 BPM (beats per minute), respectively.

To assess the validity of the experiments, we have employed the chi-square test:

$\chi^{2}=\sum_{i=1}^{k}\left(O_{i}-E_{i}\right)^{2} / E_{i}$

where $O_{i}$ is heart rate values from the PIR sensor, $E_{i}$ is heart rate values from the PPG sensor, and $k=60$. The hypothesis "estimated heart rate values from PIR sensor are reliable" is true with a significance level of $\alpha=0.05$.

\section{Conclusion}

This paper presents a PIR sensor based system capable of estimating the Resting Heart Rate (RHR). The PIR based system is an efficient and low cost option for heart rate monitoring. We estimate the RHR from the 2nd order derivative of the analog PIR sensor signal which exhibits an almost periodic behavior due to the heart beat. Therefore, it is possible to determine the RHR from the zero crossings of the second order derivative signal. The subject should be at rest during the RHR measurement process so as not to disturb the second order derivative signal, which captures the acceleration of the chest. We verified our RHR results by using a PPG sensor. We also believe that the implications of this work extend beyond the RHR estimation. Since we obtain a waveform describing the activity of the heart from the PIR sensor it may be possible to monitor heart rate variability from the zero-crossings or peaks of the second order derivative signal.

\section{References}

[1] R. McCraty, Science of Heart, Boulder Creek: HeartMath Institute, 2015.

[2] R. McCraty, Fred Shaffer, Heart rate variability: new perspectives on physiological mechanisms, assessment of self-regulatory capacity, and health risk, Global Adv. Health Med. 4 (1) (2015) 46-61.

[3] P. Kshirsagar, S. Akojwar, Classification of human emotions using EEG signals, Int. J. Comput. Appl. 146 (7) (2016) 17-23.

[4] F.M. Abuhashish Amin, H. Kolivand, M.S. Sunar, Framework of controlling 3D virtual human emotional walking using BCI, J. Teknol. (Sci. Eng.) 75 (4) (2014) $17-25$.

[5] D.S. Kim, T.H. Hwang, J.Y. Song, S.H. Park, J. Park, E.S. Yoo, N.K. Lee, J.S. Park, Design and fabrication of smart band module for measurement of temperature and GSR (galvanic skin response) from human body, in: 30th Eurosensors Conference, EUROSENSORS 2016, 2016.

[6] I. Sadek, J. Biswas, B. Abdulrazak, Z. Haihong, M. Mokhtari, Continuous and unconstrained vital signs monitoring with ballistocardiogram sensors in headrest position, in: IEEE-EMBS International Conferences on Biomedical and Health Informatics, Orlando, 2017.

[7] E. Peper, R. Harvey, I.-M. Lin, H. Tylova, D. Moss, Is there more to blood volume pulse than heart rate variability, respiratory sinus arrhythmia, and cardiorespiratory synchrony?, Assoc Appl. Psychophysiol. Biofeedback 35 (2) (2007) 54-61.

[8] Y.-P. Hsu, Z. Liu, M.M. Hella, A low-power adjustable bandwidth biomedical signals acquisition SoC for continuous health monitoring system, in: Circuits and Systems (ISCAS), 2016 IEEE International Symposium, Montreal, 2016.

[9] P. Das, A. Khasnobish, D.N. Tibarewala, Emotion recognition employing ECG and GSR signals as markers of ANS, in: Conference on Advances of Signal Processing, Pune, 2016.

[10] M.T. Jensen, P. Suadicani, H.O. Hein, F. Gyntelberg, Elevated resting heart rate physical fitness and all cause mortality, Heart (British Cardiac Society) (2013).

[11] J. Achten, A.E. Jeukendrup, Heart rate monitoring - applications and limitations, Sports Med. (2003) 517-538.

[12] G. Berndton, J. Bigger Jr., D.L. Eckberg, G. Paul, P.G. Kaufmann, M. Malik, H.N. Nagaraja, S.W. Porges, J.P. Saul, P.H. Stone, M.W.V.D. Molen, Heart Rate Variability: Origins, Methods, and Interpretive Caveats, Psychophysiology Cambridge University Press, Cambridge, 1997.

[13] H.W. Guo, Y.S. Huang, K. Haraikawa, J.C. Chein, J.S. Shieh, Short-term analysis of heart rate variability for emotion recognition using a wearable ECG device, in: ICIIBMS 2015, Track 3: Bioinfomatics, Medical Imaging, and Neuroscience, Okinawa, 2015.

[14] T. Tamura, Y. Maeda, M. Sekine, M. Yoshida, Wearable photoplethysmorgraphic sensors-past and present, Electronis-MDPI (2014) 282-302.

[15] M.E.S. Luna, S. Fullam, Piezoelectric Heart rate Sensing for Wearable Devices, San Francisco Patent US 20140128753A1, 8 May 2014.

[16] Svanberg, E. Krite, Non-invasive Optical Monitoring of Free and Bound Oxygen in Humans, Lund University, Faculty of Medicine Doctoral Dissertation Series 2016, Malm, 2016.

[17] Amy Diane Droitcour, Non-contact Measurement of Heart and respiration Rates with Single-Chip Microwave Doppler Radar, Stanford University, Stanford, 2006.

[18] D. Ghosh, P.K. Sahu, UWB in healthcare, in: 2016 International Conference Electromagnetics in Advanced Applications (ICEAA), Verona, 2016.

[19] F. Adib, H. Mao, Z. Kabelac, D. Katabi, R.C. Miller, Smart homes that monitor breathing and heart rate, in: ACM Conference on Human Factors in Computing System, Seoul, 2015.

[20] V. Hers, D. Corbugy, I. Joslet, P. Hermant, J. Dermarteau, B. Delhougne, C. Vandermoten, J.P. Hermanne, New concept using Passive Infrared (PIR) technology for a contactless detection of breathing movement: a pilot study involving a cohort of 169 adult patients, J. Clin. Monitor. Comput. 27 (2013) 521-529.

[21] J. Yun, S.-S. Lee, Human movement detection and identification using pyroelectric infrared sensors, Sensors-Open Access J. - MDPI (2014).

[22] Aron E. Suppes, Human Identification using Pyroelectric Infrared Sensors, University of Nevada, Las Vegas, 2009.

[23] X. Luo, H. Tan, Q. Guan, T. Liu, H.H. Zhuo, B. Shen, Abnormal activity detection using pyroelectric infrared sensors, Sensors-Open Access J. - MDPI (2016).

[24] A. Pantelopoulos, N.G. Bourbakis, A survey on wearable sensor-based systems for health monitoring and prognosis, IEEE Trans. Syst., Man Cybernet. (2010).

[25] C. Takano, Y. Ohta, Heart rate measurement based on time-lapse image, Med. Eng. Phys. - J. - Elsevier (2006).

[26] L. Iozzia, L. Cerina, L. Mainardi, Assessment of beat-to-beat heart rate detection method using a camera as contactless sensor, IEEE Xplore (2016) 521-524. 
[27] L.A. Aarts, V. Jeanne, J.P. Cleary, C. Lieber, J.S. Nelson, S.B. Oetomo, W. Verkruysse, Non-contact heart rate monitoring utilizing camera photoplethysmography in neonatal intensive care unit - a pilot study, Early Hum. Develop. (2013).

[28] L. Scalise, U. Morbiducci, Non-contact cardiac monitoring from carotid artery using optical vaibrocardiography, Med. Eng. Phys. - J. - Elsevier (2007).

[29] M. Uenoyama, T. Matsui, K. Yamada, S. Suzuki, B. Takase, S. Suzuki, M. Ishihaar, M. Kawakami, Non-contact respiratory monitoring system using a ceiling attached microwave antenna, Med. Biol. Eng. Comput. (MBEC) - Springer (2006) 835-840.

[30] T. Choudhary, M. Sabarimalai Manikandan, Robust Photoplethysmographic (PPG) based Biometeric Authentication for Wireless Body Area Networks for m-Health Applications, 978-1-5090-2361-5/16, 2016.

[31] M. Brink, C.H. Muller, C. Schierz, Contact-free measurement of heart rate respiration rate and body movements during sleep, Behav. Res. Models (2006) 511-521.

[32] M.Z. Poh, D.J. McDuff, R.W. Picard, Advancements in noncontact, multiparameter physiological measurements using a Webcam, IEEE Trans. Biomed. Eng. (2011) 7-11.
[33] O. Gupta, D. McDuff, R. Raskar, Real-time physiological measurement and visualization using a synchronized multi-camera system, in: IEEE Conference on Computer Vision and Pattern Recognition Workshops, 2016.

[34] P.H. Souza, J. Ferreira, T.A. Barbosa, HRVCam: A Software for Real-Time Feedback of Heart Rate and HRV, IEEE 978-1-5090-4199-2, 2016.

[35] L. Scalise, Non-contact heart monitoring, Adv. Electrocardiog. - Methods Anal. (2012).

[36] S.D. Min, J.K. Kim, H.S. Shin, Y.H. Yun, C.K. Yun, M. Lee, Noncontact respiration rate measurement system using an ultrasonic proximity sensor, IEEE Sens. J. (2010) 1732-1739.

[37] M. Zhao, F. Adib, D. Katabi, Emotion recognition using wireless signals, in: The 22nd Annual International Conference on Mobile Computing and Networking, New York City, 2016.

[38] Noise Robust Diffrentiators for Second Derivative Estimation [Online]. <http://www.holoborodko.com/pavel/downloads/ NoiseRobustSecondDerivative> (accessed 10 February 2017). 